\title{
Sociedad Femenil Rosa Luxemburgo Escuela de Derecho, Universidad de Chihuahua (1967-1968)
}

\author{
Female Society "Rosa Luxemburgo" \\ Law School, University of Chihuahua (1967-1968) \\ NITHIA CASTORENA-SÁENZ \\ Facultad de Filosofía y Letras, Universidad Autónoma de Chihuahua \\ castorenasaenz@gmail.com \\ ORCID 0000-0002-7760-0931 \\ http://dx.doi.org/10.15304/sm.31.5995
}

\section{RESUMEN}

La Sociedad Femenil Rosa Luxemburgo, fue fundada en la Escuela de Derecho de la Universidad de Chihuahua (1966) por jóvenes estudiantes identificadas con una línea política de izquierda. Algunas se encontraban adscritas con anterioridad a otro grupo político, pero decidieron formar uno exclusivo de mujeres para reivindicarse, pues de otra forma su participación era limitada. La claridad de posicionar a las mujeres y darles un papel central siempre estuvo presente en sus procesos, Las Rosas (como les llamaban) se encargaron de promover acciones políticas y de estudio, encaminadas a incrementar la participación femenina en términos más igualitarios, además de apoyar e involucrarse con la operación de grupos armados en Chihuahua entre 1965 y 1972.

Este artículo es parte de una investigación más amplia sobre la operación de los grupos armados en Chihuahua, titulada Estaban ahí. Las mujeres en los grupos armados de Chihuahua.

Palabras Clave: Guerrilla, Mujeres, Política de izquierda, 60s, Chihuahua.

\section{Abstract}

The Female Society Rosa Luxemburgo was founded in School of Law of Universidad de Chihuahua (1966) by young students identified with a left political line. Some of them were previously assigned to another political group, but decided to create an exclusive group of women to vindicate themselves, since otherwise their participation was limited. The clarity of positioning women and giving them a central role was always present in their processes. Las Rosas (as they were called) was in charge of promoting political and study actions aimed at increasing female participation in a more equal environment, in addition to supporting and get involved with the operation of armed groups in Chihuahua between 1965 and 1972.

This article is part of a larger investigation into the operation of armed groups in Chihuahua, entitled Estaban ahí. Las mujeres en los grupos armados de Chihuahua.

Keywords: Guerilla, Woman, Left Politics, 60's, Chihuahua. 
Libertad es siempre la libertad de quien piensa distinto

R. Luxemburgo

\section{INTRODUCCIÓN}

Aquí se propone que no es posible escribir, analizar, desarrollar y/o pensar desde otro lugar que no sea el propio, por lo que es necesario contribuir a las múltiples y variadas historias regionales, en el ánimo de una comprensión más general de lo que fue el pasado contemporáneo de México, aquel que involucró tanto los movimientos sociales, estudiantiles y, finalmente, el movimiento armado mexicano. Necesariamente este planteamiento implica la generación de nuevas preguntas y, por ende, a la construcción de explicaciones diversas de un pasado que no es otra cosa que colectivo.

La Sociedad Femenil Rosa Luxemburgo que se fundó y operó en la escuela de Derecho de la Universidad de Chihuahua, es un ejemplo de las peculiaridades locales (y localizadas) que es necesario reconstruir para comprender tanto el ayer social y colectivo, así como una reconstrucción de las formas específicas en las que las mujeres se vieron involucradas en estos procesos y, como en este caso, fueron además punta de lanza. Es oportuno precisar que no hay otro antecedente escrito que aborde de forma exclusiva el surgimiento y operación de Las Rosas, como se les conocía entre el estudiantado.

Cada país y, específicamente, cada estado de la república "cocinó" de una forma singular su propio 1968. Esta conclusión implica la premisa que guía la reconstrucción que aquí se comparte: aunque hay una evidente dimensión global de 1968, ésta no surgió como parte de un proceso organizativo también global, sino que fue producto de procesos propios, regionales, en respuesta a prácticas autoritarias de los gobiernos nacionales o locales, en contubernio con el brazo capital de cada lugar. Es claro que aún queda mucho por aprehender y comprender de un 1968 global, y esto no será posible lograrlo a partir de historias que pretendan ser generales o enfocadas en "actores principales", sino que se logrará a partir de las historias regionalizadas, entendidas en su propia dimensión y contexto, cada una con sus actores particulares.

Esto implica además reconocer que son más las coincidencias que se pueden encontrar en las prácticas de los poderes autoritarios, sobre todo las ya conocidas que se entretejen y operan a través de los brazos del poder político y económico (en ambos casos a través de fuerzas armadas estatales -militares o no- y paramilitares); mientras que las resistencias se distinguen por sus diversidades, que aún tienen mucho que mostrar.

Por último, se propone una estrategia para que este pasado contemporáneo de Chihuahua sea aprehendido no sólo a través de la comprensión y la urdimbre de eventos y acciones, sino a través de la piel: es preciso reconstruir el amor, todas las expresiones del amor, de las relaciones amorosas. 


\section{BREVE CRONOLOGÍA}

El caso de Chihuahua es paradigmático. Podría decirse que el 1968 chihuahuense se "empezó" a cocinar en noviembre de 1959, cuando fue asesinado Francisco Luján Adame, líder social en el municipio de Madera. Región con una experiencia de lucha agraria amplia, que luego desembocaría en el ya conocido asalto al cuartel de Madera en 1965. Sin embargo, el asesinato de Luján Adame no es un hecho aislado, después de éste, se creó en 1960 la Federación de Obreros y Campesinos de Chihuahua. En abril de 1961, se lleva a cabo un mitin de apoyo a la Revolución cubana en la capital chihuahuense, convocado por organizaciones ligadas al Movimiento de Liberación Nacional, en el que participaron varios estudiantes. ${ }^{1}$

Dos años después se realizó el Primer Encuentro de la Sierra en Cebadilla de Dolores, Madera. El 6 de Abril de 1964 estudiantes y simpatizantes de los grupos campesinos, entre ellos José Refugio Molina, José Mariñelarena, Víctor Zavala, y Óscar Sandoval, intentaron tener una audiencia con el entonces candidato Gustavo Díaz Ordaz, durante su gira de campaña en Chihuahua, pero éste recibiría sólo a Mariñelarena, y en un mal entendido, algunos participantes creyeron que se lo llevaban detenido, se encendieron los ánimos y el evento se salió de control, culminando en la quema del templete que se le había preparado frente a la presidencia municipal, finalizando con su salida urgente del lugar y con detenciones (Viramontes, 2009). En uno de los periódicos describen que «Ayudantes personales del candidato pretendieron calmar a la turba y sólo recibieron proyectiles que les causaron heridas leves. Mariñelarena salió a las puertas del hotel para pretender calmar a los presentes y desde el capacete de una patrulla hizo esfuerzos por hablarles, pero nada consiguió» ${ }^{2}$ (El Universal en Centro de Estudios Nacionales, S.C., 1964: 268-269). Ese mismo día hubo detenidos del movimiento estudiantil y campesino por su participación en dicho evento, algunos fueron liberados varios meses después, como Jorge Rodas y Álvaro Ríos, cuya liberación se da hasta noviembre de ese año (García Aguirre, 2012: 180-181).

1 Entre ellos, Víctor Orozco Orozco, un personaje central del movimiento estudiantil chihuahuense de entonces, y un respetado líder social y académico, menciona que luego de este mitin, varias personas protestaron contra el Periódico El Heraldo, que apenas si había cubierto la nota respecto a dicho mitin.

2 Sobre esta publicación es oportuno mencionar que ésta se limita a transcribir, con todo y sus faltas de precisión, las notas periodísticas de diarios nacionales respecto a los acontecimientos de la campaña de GDO por estos estados. Incluso, se encuentran en esta publicación notas contradictorias o que omiten información, lo que sugiere que no tuvo, en lo más mínimo, un trabajo de análisis, sino que se limitó de manera estricta a una transcripción. Como investigadora de estos temas, no se puede hacer otra cosa que agradecer la información acumulada y dispuesta en un solo texto, lista para ser analizada. En esta misma nota se menciona que el gobernador de Chihuahua, Praxedes Giner Durán «reclamó por qué no se había hecho uso de la fuerza pública para detener el motín», luego de la explicación por parte de la judicial, de que habían recibido demasiado tarde el llamado, éste lamentó los hechos y advirtió que se castigaría a los responsables, y que dicho motín «no era obra de panistas» porque «inclusive los panistas honrados apoyan la candidatura del licenciado Díaz Ordaz.» Por otro lado, llama la atención que este mismo periódico, en esta misma fecha de publicación, incluya otras notas sueltas con titulares como «Cálido recibimiento al candidato del PRI.» 
Los periódicos a nivel nacional no mencionaron en absoluto el incidente en Chihuahua, al contrario, exaltaron el discurso diazordacista en cuanto a la justicia en el campo, «Nada justifica la invasión de las tierras de los tarahumaras» (El Día en Centro de Estudios Nacionales, S.C., 1963:264); «Díaz Ordaz promete a los tarahumaras devolverles lo que es suyo» (Últimas Noticias en Centro de Estudios Nacionales, S.C., 1963: 265). El periódico local El Heraldo de Chihuahua, no mencionó la quema del templete y, al contrario, al día siguiente publicó varias inserciones pagadas que lo felicitaban y saludaban. ${ }^{3}$ En otro más de los periódicos se mencionó que el candidato «estuvo acompañado por guapas y hermosas jóvenes de la localidad» (El Día en Centro de Estudios Nacionales, S.C., 1963: 264).

Todas estas acciones configuraron un ambiente suficientemente político que exigía posicionarse claramente frente a un gobierno autoritario, despótico, que no sólo omitió actuar bajo principios básicos de justicia y transparencia, sino que hizo gala de su fuerza en contra de la población que debería defender. Lo que parecieron entonces movimientos sociales agrarios aislados en las zonas rurales del estado de Chihuahua, no pasaron inadvertidos para la población en las zonas urbanas.

En 1965 se llevó a cabo el Segundo Encuentro de la Sierra "Heraclio Bernal", en Torreón de Cañas, Durango. En este es evidente el hartazgo por no obtener soluciones a su problemática a través de acciones en las instancias de justicia correspondientes. Se propone la lucha armada, lo que crea una escisión entre los participantes, entre aquellos que no veían las armas como una opción y quienes, meses después, en la madrugada del 23 de septiembre, ejecutaron el asalto al cuartel de Madera (acción en la que mueren 8 guerrilleros).

En febrero de 1966, el Movimiento 23 de septiembre organiza un ataque a las vías del tren a la altura de Gosogachi. En enero de 1967, la Dirección Federal de Seguridad (DFS) detiene a prácticamente todos los miembros del Movimiento 23 de septiembre, en su casa de seguridad en el entonces Distrito Federal. En ese mismo año Óscar González Eguiarte comienza con la conformación del Grupo Popular Guerrillero “Arturo Gámiz”.

3 Entre las inserciones, pueden encontrarse las del: Sindicato de Trabajadores de los Poderes del Estado de Chihuahua, Funcionarios, técnicos, empleados y trabajadores de Celulosa de Chihuahua, S.A.; Unión Ganadera Regional de Chihuahua; Asociación Minera de Chihuahua; Asociación de Mineros de Nuevo León, A.C.; Asociación de Mineros de Coahuila y Durango, A.C.; Mineros Independientes de Tamaulipas; Sindicato Nacional de Trabajadores de Aeronaves de México; el Grupo de tamaulipecos radicados en el estado de Chihuahua; Confederación de Asociaciones Locales de Productores de Manzana del Estado de Chihuahua; Banco Comercial Mexicano S.A.; Sindicato Nacional de Trabajadores del Seguro Social, Sección VIII; personal de pagadores de la Secretaría de Hacienda y Crédito Público en el Estado de Chihuahua; la Sección Número 30 del Sindicato Industrial de Trabajadores Mineros, Metalúrgicos y Similares de la República Mexicana, de Naica; Banco Provincial del Norte, S.A.; Rodolfo Porras Molinar; Unión de Productores e Industriales Forestales de Chihuahua, A.C.; Plywood Ponderosa de México, S.A.; Viscosa de Chihuahua, S.A. El número y la importancia de estas empresas y sindicatos, no dejan lugar a dudas del grado de estrechés en las relaciones entre la iniciativa privada, sindicatos y gobierno federal, una misma clase. 
En la Preparatoria de la Universidad de Chihuahua se conformó y operó, entre 1968 y 1969, el Frente Revolucionario Estudiantil Preparatoriano ${ }^{4}$, el cual aglutinó entre sus integrantes a personas que luego decidieron integrarse en varios de los grupos armados de Chihuahua. ${ }^{5}$

Junio de 1968, el Grupo Popular Guerrillero “Arturo Gámiz” quema un aserradero en Tomochi, en respuesta a las injusticias que éste cometía contra la población; la operación es bautizada como "Miguel Quiñones". En septiembre de ese mismo año, este grupo es aniquilado por el ejército entre la sierra de Chihuahua y la de Sonora.

\section{LA UNIVERSIDAD DE CHIHUAHUA: UN CATALIZADOR}

La Universidad de Chihuahua se fundó en 1954 por un decreto promovido ante el Congreso del Estado por el entonces gobernador, Óscar Soto Maynez. Ésta se instala entonces en el espacio del Instituto Científico y Literario de Chihuahua, en el mismo edificio que ahora ocupa la Rectoría de dicha Universidad (Decreto No. 171, 1954).

Según su página oficial en sus primeros años dio servicio a una población escolar que apenas alcanzaba un millar de personas inscritas cada ciclo escolar. En aquel entonces, la matrícula total de Educación Superior en el país estuvo constituida por 29 mil estudiantes. La Universidad comenzó con las escuelas de Medicina, Ingeniería, Derecho, Educaciónn física y Farmacia.

Frente a la escuela de Derecho se encontraban las instalaciones de la Escuela Normal del Estado, atrás la Escuela de Ingeniería y junto a ésta la Preparatoria de la misma Universidad. Se sugiere muy posible que esta vecindad espacial haya sido una más de las circunstancias que facilitaron el intercambio entre las personas que realizaron sus estudios en estas escuelas.

La Preparatoria de la Universidad se formó de lo que se había conocido como el Instituto Científico y Literario, espacio educativo reconocido por una tradición liberal, inspirada sobre todo en el legado juarista ${ }^{6}$. Dado que la oferta educativa de nivel medio superior estuvo concentrada en la capital, además de ser la única posibilidad para educación profesional, la ciudad de Chihuahua se estableció como una especie de centro estudiantil natural, Víctor Orozco menciona que: «Chihuahua era un embudo, llegábamos de todo el estado» ${ }^{7}$.

4 Entrevista a Carmen Monares, realizada por Nithia Castorena-Sáenz (en Adelante NCS) el 4 de mayo de 2013.

5 En entrevista, Juan Chávez, quien fue integrante del Movimiento de Acción Revolucionaria, mencionó que a él lo invitaron tres veces a participar en diferentes grupos armados. Todas las invitaciones ocurrieron en la escuela y fueron de gente que ya conocía su participación en la política estudiantil de la preparatoria.

6 Víctor Orozco menciona incluso, que era un liberalismo radical para esa época y que fue en la tradición en la que él fue formado.

7 Entrevista a Víctor Orozco Orozco realizada por NCS el 3 de abril de 2013, Ciudad Juárez, Chihuahua. 
Es claro que la Universidad no permaneció ajena a las cuestiones sociales que la rodearon. Una de las características que tuvo fue su apertura a influencias externas. En sus espacios fue posible dar seguimiento, comentar y compartir puntos de opinión respecto a los hechos de índole nacional e internacional incluso, que ocurrieron en esa época. La mayoría de las personas entrevistadas mencionaron como una gran influencia a las personas que fueron sus maestros en la Preparatoria de la Universidad, entre éstos Ernesto Lugo, Olac Fuentes Molinar, Federico Ferro Gay y Antonio Becerra. ${ }^{8}$ Además fueron muy populares los concursos de oratoria que se organizaron, sobre todo en la Escuela de Derecho. Y es notoria la relación estrecha que hubo entre las sociedades de alumnos de las distintas escuelas, lo que facilitó, por ejemplo, que se conocieran Rubén Aguilar y Diego Lucero cuando ambos fueron presidentes de las sociedades de alumnos de la Escuela de Derecho y de la de Ingeniería, respectivamente, ${ }^{9}$ relaciones y personajes que años después impactarían en la lucha social del estado de Chihuahua.

En cuanto al crecimiento institucional y de oferta de formación profesional, la Universidad creció de forma inmediata una vez inaugurada. En septiembre de 1956 fue fundada la Escuela de Ganadería, hoy Facultad de Zootecnia. La Escuela de Contabilidad y Administración fue creada el 3 de diciembre de 1958, como Escuela de Comercio y Administración, hoy Facultad de Contaduría y Administración. En 1960 se fundó la Biblioteca Central con 2 mil 353 volúmenes. En julio de 1963 se fundó la Escuela de Filosofía, Letras y Periodismo. El 22 de julio de 1967, la Universidad funda la Escuela de Agronomía, la cual es trasladada después a Delicias.

Para 1968, durante el gobierno de Óscar Flores, la Universidad adquirió su autonomía y se transformó en Universidad Autónoma de Chihuahua. En este mismo año se fundó la Escuela de Administración Pública y Ciencias Políticas, en el campus de Ciudad Juárez.

Una cuestión significativa es el hecho de que Chihuahua tuvo, de 1950 a 1960, la mayor tasa de crecimiento de población de todo el siglo XX (INEGI, 2006:17) ${ }^{10}$. Es decir, el estado nunca ha crecido tanto, en cuanto a número de habitantes como durante esa década, lo que implica que para los años posteriores a 1960 y entrada la década de 1970, la educación fue una necesidad imperante para toda esa población infantil y joven. La migración de las zonas rurales a las urbanas, sobre todo a la capital del estado, se debió en gran parte a las opciones educativas y de empleo que ahí se encontraron.

8 Este último, secuestrado en 1969, lo que generó una gran movilización de parte de estudiantes, logrando que fuera liberado. Seguramente este hecho contribuyó a reafirmar en éstos el poder político que tenían.

9 Entrevista a Jaime García Chávez realizada por NCS el 7 de abril de 2013.

10 Este documento describe la Tasa media anual de crecimiento como «la velocidad o ritmo de cambio de los efectivos de la población en un periodo determinado. La tasa se obtiene al relacionar una población en dos momentos, asumiendo un modelo de comportamiento, en este caso geométrico, lo que supone el incremento acumulado de la población en función de la población inicial.» (INEGI, 2006: 293). Además, estas cifras coinciden con las encontradas a nivel internacional, para las que en 1960 es el punto más alto en cuanto a la "Tasa de crecimiento demográfico mundial". Para más información al respecto puede consultarse (Demografía, 18-24 de junio de 1981). 
De 1960 a principios de 1970, en general, el estado de Chihuahua tuvo poca población. ${ }^{11}$ Las cifras que manejan los censos de la época sugieren una ciudad capital en la que las familias debieron conocerse entre sí, con pocos barrios, distancias de recorridos locales cortas, por lo que los espacios de interrelación social ocurrieron necesariamente en las rutinas diarias, ya sea la escuela, la tienda, el mercado, las calles del barrio o algún parque. Este detalle pareciera una cosa menor a no ser porque una conclusión recurrente de las organizaciones sociales en la década de 1960, sobre todo campesinas y estudiantiles, ${ }^{12}$ fue la inminencia de llevar a cabo acciones armadas, que no tenían otra forma de desarrollarse que de forma clandestina y: ¿qué tan clandestina puede ser la “organización clandestina" cuando las rutinas cotidianas, en la ciudad de Chihuahua, no propiciaban otra cosa que la convivencia? ${ }^{13}$

Es importante anotar que la migración de las zonas rurales a las ciudades creció en este periodo. La crisis del campo mexicano es un proceso complejo y dicha complejidad ya era evidente para la década de 1960. Entre otras causas, es posible identificar la falta de créditos y apoyo para la tecnificación en el ramo agrícola, así como el desentendimiento del gobierno en cuanto a la proliferación de oligarcas y terratenientes. Todas estas condiciones derivaron en un detrimento de la calidad de vida de las personas de las zonas rurales, lo que contribuye a explicar una de las razones de su migración hacia las ciudades. Sin embargo también es importante precisar que ésta no siempre se vio forzada por las condiciones de marginación y rezago del trabajo en el campo, sino por la búsqueda de opciones de desarrollo educativo para la familia. Es posible ver este supuesto reflejado en el hecho de que la mayoría de las personas integrantes de grupos políticos al interior de la Universidad de Chihuahua, en específico de la Escuela de Derecho, provenían de municipios distintos a la capital y llegaron a ésta sin más objetivo que la continuación de sus estudios. ${ }^{14}$

11 Representó en esa década, alrededor del 3\% de la población del país, mientras que el Distrito Federal representó, por ejemplo, alrededor del $11 \%$.

$12 \mathrm{Al}$ respecto de la intensa relación entre organizaciones sociales y estudiantiles, véase: García Aguirre, 2012.

13 Desde 1950 hasta 1970 Chihuahua representa alrededor del 3\% de la población del país. El porcentaje de mujeres con respecto al de hombres guarda la misma proporción que a nivel nacional y que los estados colindantes, poco más del $49 \%$ de mujeres, frente a poco más del $50 \%$ de hombres. En cuanto al hecho de utilizar a hijas e hijos de conocidos para enviarles recados a éstos, o el hecho de encontrarse diariamente a personas conocidas, las referencias se encuentran en: Entrevista a Marco Rascón Córdoba, realizada el 11 de enero del 2012 en Chihuahua, Chihuahua; y Entrevista a Carlos Fernández Baca, realizada el 23 de febrero del 2013, en Chihuahua, Chihuahua.

14 Las opciones educativas en la capital del estado durante la década de 1960 fueron: el Instituto Palmore, con primaria y secundaria; la Escuela de Artes y Oficios (Ésta escuela cerró en 1964, lo que provocó una huelga entre varias escuelas chihuahuenses inconformes con esta decisión. Para más información al respecto puede consultarse la tesis de García Aguirre, 2012. El Colegio Regional con secundaria y preparatoria, administrado por la Compañía de Jesús en Chihuahua (Entrevista realizada a Víctor Orozco Orozco el 3 de abril del 2013 en Ciudad Juárez, Chihuahua); y por último, la Universidad de Chihuahua (UCh) y su escuela preparatoria. Además de ésta, era posible desarrollar estudios profesionales en el 
En el caso del primer grupo armado de Chihuahua, que además fue el primero a nivel nacional en la segunda mitad del siglo XX: el Grupo Popular Guerrillero, ${ }^{15}$ sus bases de apoyo urbanas estuvieron compuestas sobre todo de estudiantes, tanto de la Escuela de Derecho como de la Escuela Normal de Saucillo. Para ser una especie de subgrupo de las organizaciones armadas, los y las estudiantes eran una mayoría dentro de los mismos; sin embargo, en absoluto representaron la generalidad del estudiantado en Chihuahua. ${ }^{16}$

Si bien la condición de estudiante por sí sola no definió la participación en grupos armados, la condición de joven fue lo más cercano a un común denominador, aunque tampoco fue la regla. ${ }^{17}$ En este sentido se vuelve trascendente la cantidad de jóvenes que hubo en México en la época de estudio. En un rango de edad desde los 10 hasta los 29 años, esta "ola juvenil" se acercaba al $40 \%$ del total de población. La proporción se mantuvo desde 1960 hasta $1970 .^{18}$

El hecho de que las mujeres no aparezcan en la mayoría de los criterios de los censos que se han citado, no implica per sé una condición de desventaja en este periodo. Ese no puede ser el resultado de un análisis sino una condición conocida de la que es posible aprehender al observarse desde la perspectiva de género y de la historia social: los sujetos sin poder rara vez aparecen en la narrativa del pasado, sobre todo en la de las instituciones. Lo que busco plasmar son las formas en que las mujeres resistieron el ejercicio de poder en contra de ellas. Propongo que una de las resistencias esenciales de las mujeres se encuentra en las actividades públicas y políticas que desarrollaban, como en este caso, a través de la Sociedad Femenil Rosa Luxemburgo.

\section{LAS ROSAS: SOCIEDAD FEMENIL ROSA LUXEMBURGO}

Este grupo, constituido en la Escuela de Derecho (UCh) en 1966, toma como figura representativa la de la teórica marxista Rosa Luxemburgo. Se considera preciso contextualizar de forma breve las luchas contemporáneas emprendidas entonces por las mujeres pues, a pesar de su agudeza analítica y crítica, sus escritos no fueron dedicados exclusi-

Instituto Tecnológico de Chihuahua, activo desde 1948, o en la Escuela Normal, o la Escuela Normal Superior, entonces dependientes ambas del Gobierno del Estado de Chihuahua.

15 Cuya última acción fue el asalto al Cuartel de Madera el 23 de septiembre de 1965.

16 La media nacional, para las profesiones "sociales" -entre las que la Dirección General de Estadística (DGE) consideraba Derecho y Economía- se encontró en 32.02\%; Chihuahua por su parte estuvo por abajo de este porcentaje, con un $21.15 \%$. El porcentaje más alto de los estados colindantes con Chihuahua lo tuvo Sonora con un $40.97 \%$, casi a la par de sus estudiantes de nivel profesional en el área técnica.

17 El caso de los doctores Pablo Gómez y Napoleón Glockner, son algunas de las excepciones, pues ambos eran padres de familias numerosas y mayores de treinta años, al momento de involucrarse en grupos armados, el primero en el Grupo Popular Guerrillero, en Chihuahua; el segundo en las Fuerzas de Liberación Nacional, en Monterrey.

18 Se consideran jóvenes a partir de los 10 años pues se toman los datos del censo que se llevó a cabo en 1960, para mediados de la década estos niños ya se encontrarían entrando en la edad adolescente (Dirección General de Estadística, 1963). 
vamente a la condición de las mujeres dentro de su partido (Partido Social Demócrata del Reino de Polonia), por ejemplo. ${ }^{19}$ Es factible considerar que si ella estuvo en condiciones de ser una de las principales colaboradoras del periódico teórico marxista más importante de la época, Die Neue Zeit, fue debido a los triunfos de las luchas por el derecho de las mujeres a la participación política. A Luxemburgo le anteceden la Déclaration des Droits de la Femme et de la Citoyenne (Declaración de los Derechos de la Mujer y la Ciudadana), redactado en 1791 por Olympe De Gouges en alusión a la Declaración de Derechos del Hombre y del Ciudadano del 26 de agosto de 1789, emanada de la Revolución Francesa. ${ }^{20} \mathrm{Al}$ año, en Inglaterra se publica la "Reivindicación de los Derechos de la Mujer" de Mary Wollstonecraft. Luego, para 1848, se lleva a cabo la primera Woman's Rights Convention en Seneca Falls, Nueva York. La segunda se celebra en Worcester, Massachusetts, en 1851. ${ }^{21}$ Es en este contexto que una mujer como Rosa Luxemburgo, judía, joven, con una leve discapacidad en una pierna, estudia, reflexiona, viaja a otro país para continuar con sus estudios y se integra con entusiasmo en la política de izquierda, contribuyendo no sólo con textos y debates, sino con su cuerpo y su fuerza, ${ }^{22}$ por lograr aquello en lo que creía.

Integrado sólo por mujeres, el grupo se distingue por haber sido fundado por jóvenes estudiantes identificadas con una línea política de izquierda, algunas de ellas adscritas con anterioridad a la Sociedad Ignacio Ramírez (Los Nachos) que operó de forma paralela en la misma Escuela. Cuenta Cristina González Tejeda:

Yo fui nacha. Sí nos dejaron entrar, como éramos muchas, pues hasta nomás por estarnos viendo, en ese entonces se usaba la minifalda, y éramos muy guapas, y fumábamos en público, cuando fumar era una gracia, entonces claro que nos dejaron entrar a Los Nachos. ${ }^{23}$

La perspectiva crítica de la Sociedad Femenil Rosa Luxemburgo es evidente desde el momento en que disintieron de la Sociedad Ignacio Ramírez, a la que estuvieron integradas previamente; sin embargo, este ejercicio crítico no surgió de una condición vivida (experimentada en ellas mismas) de marginación económica; ser estudiantes de la

19 Para abundar más en la vida de esta teórica se puede consultar Dunayevskaya, R., 1985.

20 A causa de este escrito, De Gouges fue condenada a la guillotina por el incipiente cambio de poderes del momento, un tribunal revolucionario. Ni siquiera sus colegas revolucionarios, ansiosos de cambiar un régimen monárquico, por antiguo e injusto, fueron sensibles a la condición de las mujeres francesas.

21 En ella se hacen públicos los saludos de Jeanne Deroin y Pauline Roland, francesas detenidas por sus actividades en la revolución de 1848.

22 Al puntualizar que es con su cuerpo y su fuerza, se está posicionando teórica y epistemológicamente, desde el feminismo. La localización, que implica tanto el cuerpo como la experiencia de las mujeres, es una postura ya muy socorrida desde hace muchos años por las corrientes feministas (Monique Wittig, Gloria Anzaldúa, Cherrie Moraga, Paul B. Preciado). Además, para el caso concreto de Rosa Luxemburgo, entre otras iniciativas que llevó a cabo, trabajó en una mina de carbón para tratar de incidir en la organización de los trabajadores.

23 Entrevista a Cristina González Tejeda realizada por NCS el 23 de abril de 2013 en Chihuahua, Chihuahua. 
Escuela de derecho en una época en que no era común realizar estudios de licenciatura, da cuenta de ello. Más aún que fueran mujeres en dicha escuela, pues su población estudiantil se componía sobre todo de hombres. ${ }^{24}$

Las jóvenes que formaron la Sociedad Femenil Rosa Luxemburgo (Las Rosas) se encargaron de promover acciones políticas y de estudio alrededor de una ideología de izquierda, al igual que Los Nachos, pero lo hicieron en el ánimo de promover la participación de las mujeres en un ámbito más igualitario. Y, como se verá a continuación, lo lograron.

Por lo que se rescata de las entrevistas realizadas, Las Rosas desconocían la trayectoria de Rosa Luxemburgo, Cristina González menciona que: «Nunca supimos en general, ni la biografía de Rosa Luxemburgo [...] era tan inicial la cosa.» ${ }^{25}$ Se sabe que el nombre fue propuesto por Irma Campos, y tanto Cristina González Tejeda, como Jaime García Chávez y Cecilia Wong Ordoñez coinciden en que el Lic. Ernesto Lugo fue una influencia importante para los grupos de izquierda en la Escuela de Derecho, e incluso García Chávez propone que tal vez él le propuso el nombre a Irma.

La Sociedad Femenil Rosa Luxemburgo tuvo carisma entre el estudiantado de la Escuela de Derecho. Las estudiantes que la formaron eran egresadas del Instituto Femenino, del Colegio América y del Colegio Chihuahuense, colegios con formación eminentemente católica, «pero que llegan a un punto en el que, el reclamo social, los hace aportar lo que en ese momento tenían, que era una ideología, y salirse de los patrones.» ${ }^{26}$

El grupo fue débil en cuanto a planteamientos feministas. La claridad de posicionar a las mujeres, darles un papel central, siempre estuvo presente en sus procesos, que no en los contenidos. Campos Madrigal menciona que las fundadoras deciden integrarse en un grupo exclusivo de mujeres por una cuestión de reivindicación, «Ya que en los Nachos casi no se tomaba en cuenta la situación de las mujeres y tampoco a nosotras se nos reconocía el liderazgo». ${ }^{27}$

Su evento de presentación fue una «Serie de Conferencias» sobre «problemas filosóficos, económicos y políticos contemporáneos» ${ }^{28}$, llevadas a cabo el 20 de febrero de 1967, lo que indica que su conformación como grupo debió ocurrir entre septiembre de 1966, fecha en que Cristina González Tejeda y Avelina Gallegos Gallegos ingresan a la Escuela de Derecho, y ese febrero de 1967. Entre sus conferencistas estuvieron sólo hombres.

24 Cristina González cuenta que cuando ella entró a la escuela de derecho (1966), ésta sólo tenía 5 alumnas y 195 alumnos hombres.

25 Entrevista a Cristina González Tejeda realizada por NCS el 23 de abril de 2013 en Chihuahua, Chihuahua.

26 Entrevista a Carlos Fernández Baca realizada por NCS el 23 de febrero de 2013, en Chihuahua, Chihuahua.

27 Entrevista a Irma Campos Madrigal realizada por Nithia Castorena Sáenz el 14 de octubre del 2008.

28 Prof. Alberto Sáenz, Sr. Jaime García Ch., Prof. Antonio Becerra, Prof. José Luis Orozco, Prof. Olac Fuentes M., Volante de La Sociedad Femenil de la Escuela de Derecho, disponible en el archivo personal de Nithia Castorena Sáenz, donado por Cristina González Tejeda. 
No hubo conferencistas mujeres, ni los temas que se abordaron fueron relacionados con la condición de las mujeres. ¿Qué hacía de Las Rosas un grupo de reivindicación entonces? Precisamente su actuación. Fueron sólo mujeres las que lo componían y para ellas ese hecho constituyó un proceso de aprendizaje. De ese modo constataron que ellas eran capaces, al igual que los hombres en Los Nachos, de llevar a cabo acciones políticas al interior de la Escuela de Derecho, y extenderlas no sólo a otros espacios universitarios, sino a un activismo constante que con objetivos estudiantiles y de apoyo a las luchas rurales y campesinas. Además, Las Rosas instalaban cada semana un periódico mural con temas de interés y análisis de noticias actuales, lo que deja manifiesta la rigurosidad y disposición de las integrantes del grupo, pues sólo de ese modo sería posible mantener una publicación semanal. ${ }^{29}$

Sobre la recepción que tuvo la Sociedad Femenil Rosa Luxemburgo en la Escuela de Derecho, Irma Campos Madrigal cuenta que fueron objeto de burlas. Durante una asamblea general a la que convocaron para presentar el grupo, ella dio el saludo de bienvenida y al saludar «Buenas tardes», todos contestaron a coro «buenas tardes». Después de eso, les preguntaban en tono de burla « $A$ Ay!, ¿ a poco eres de las rosas? $»^{30}$.

Suman varios factores los que posibilitan y fomentan la formación de Las Rosas. El primero, la determinación de sus lideresas, con algo de experiencia en la política estudiantil a partir de su participación con la Sociedad Ignacio Ramírez. Además, el hecho de que no hayan podido destacar en su liderazgo dentro de grupos masculinos, y por último, un factor muy importante, el hecho del trato diferenciado que recibían en la Escuela de Derecho. A decir de Irma, en ese entonces en la Escuela el estudiantado ascendía a más de 500 personas, de las cuales apenas 14 eran mujeres. Su condición minoritaria fue abrumante, lo que vuelve aún más fascinante a este grupo, pues no sólo resistió el embate institucional, en voz del entonces director, Augusto Martínez Gil, el cual las retaba a permanecer en la escuela y promovía que los maestros fueran más estrictos con las alumnas $\mathrm{y}$, de ser posible, las reprobaran. ${ }^{31}$

El 8 de marzo de 1967, las integrantes de Las Rosas se juntan para desayunar y conmemorar así el Día Internacional de la Mujer. Sobre este evento aparece una fotografía ${ }^{32}$ en El Heraldo de Chihuahua: «Con motivo de la celebración del 'Día de la Mujer', las alumnas de la Escuela de Derecho de la Universidad de Chihuahua, se reunieron ayer

29 Esto lo afirma Cristina González Tejeda, además de advertir lo caro que era mantener el periódico, pues había que tener cartulinas, pegamento y demás materiales para su instalación y cambio cada semana. Entrevista a Cristina González Tejeda, realizada por Nithia Castorena Sáenz el 23 de abril del 2013 en Chihuahua, Chihuahua.

30 Entrevista a Irma Campos Madrigal realizada por NCS el 14 de octubre de 2008.

31 Entrevista a Cristina González Tejeda realizada por NCS el 3 de mayo de 2013 en Chihuahua, Chihuahua.

32 En la fotografía aparecen: Cristina González, Virginia Terrazas, Martha Fierro, Irma Campos, Blanca I. Ornelas, María Luisa Pérez, Yolanda Martínez, Gloria Carrasco, Virgina Ramos, María del Socorro García, Tina Rubio, Cecilia W. de Maspulez, Elisa Mendoza, Emma Cervantes, Norma Jiménez, Norma Ordoñez, Cecilia Millán y Avelina Gallegos. 
en céntrico Restaurante, para festejarlo» (9 marzo de 1967). La nota apareció con tintes sociales, como anunciar una despedida de soltera, o una reunión familiar, incluso entrecomillado el 'Día de la Mujer'.

Sergio Granados Pineda recuerda que cuando él dirigió su campaña para presidente de la Sociedad de Alumnos, en 1966, "hubo un levantamiento" de quienes se conformarían después como Las Rosas, pues estaban en contra de que hubiera una "secretaría de la mujer" se propuso "como innovación", por parte de su planilla. Asegura que Irma Campos le dijo «oye, pues pon una secretaría del hombre ¿no? No, aquí valemos por lo que somos y podemos ocupar todos los lugares, y quita eso que denigra a las mujeres.» ${ }^{33}$ Esto pone de manifiesto el involucramiento en todas las cuestiones de la vida estudiantil que tuvieron las integrantes de Las Rosas.

Aunque Las Rosas no fueron un grupo de feministas, sí fueron un grupo de mujeres que tomaron el poder sobre sus acciones. Su condición de estudiantes en la Escuela de Derecho, cuando el director de la misma se negaba a que las mujeres estudiaran ahí, es muestra de una resistencia a los estereotipos que se les pretendieron imponer en esa época. Si bien no fue un grupo en el que todas sus integrantes se identificaran con ideas de izquierda, pues incluso algunas fueron afines al PRI, hubo varias integrantes que luego apoyaron o participaron en las acciones de los grupos armados, como Irma Campos Madrigal, Francisca Urías Hermosillo, Cristina González Tejeda y Avelina Gallegos Gallegos.

El caso de Avelina Gallegos es paradigmático pues de Las Rosas, fue la única que murió en medio de una acción armada. Carmen Monares recuerda que en algún momento entre 1969 y 1970, Avelina las invitó a ella y a Ramona Gómez a una casa ubicada en la calle Morelos, para hablar de la posible reorganización de Las Rosas: «ella muy apasionada en el tema....alimentando que hubiera una organización de mujeres de izquierda... como habían sido Las Rosas en un tiempo». ${ }^{34}$ Como la misma Carmen lo mencionó durante la entrevista, es probable que su verdadera intención no fuera la reorganización de Las Rosas sino la integración de ellas en el grupo armado que se estaba gestando, y que organizaría el triple asalto bancario del 15 de enero de 1972, en el que la misma Avelina perdería la vida.

\section{ALGUNAS IDEAS A MODO DE CONCLUSIONES}

Tal como ocurrió en el caso de Rosa Luxemburgo, su situación de conciencia social y política no se derivaba de una posición de marginación económica, sino que fue precisamente su posición no marginal, lo que les permitió (a ella y a las integrantes de la Sociedad Femenil Rosa Luxemburgo) estudiar, crear y desarrollar una posición política crítica y propia.

33 Entrevista a Sergio Granados Pineda realizada por NCS el 2 de abril de 2013 en Chihuahua, Chihuahua.

34 Entrevista a Carmen Monares realizada por NCS el 4 de mayo de 2013. 
Queda fuera de este artículo una gran parte documentada en relación al crecimiento poblacional de la ciudad de Chihuahua que logra contextualizar y dimensionar las implicaciones de integrarse a una organización o movimiento estudiantil en una ciudad pequeña en la que es común encontrarse a diario con personas conocidas. En las entrevistas que realicé para el trabajo del que se desprende este artículo, fueron recurrentes los comentarios respecto a que las invitaciones a participar en grupos armados se repetían, es decir, se intentaba reclutar a los mismos sujetos pues se identificaban con facilidad sus posiciones políticas ${ }^{35}$.

Rosa Luxemburgo fue una figura bastante lejana de la realidad de las estudiantes que conformaron Las Rosas en la Escuela de Derecho. Sin embargo tienen en común su juventud, la posibilidad de estudiar y su capacidad para sentir la injusticia experimentada por la población a su alrededor. Se propone que un análisis, poco explorado dada su compleja subjetividad, se encuentra en las relaciones amorosas que las mujeres entablaron en estos periodos de su vida ¿Qué fuentes podrían dar cuenta de esto? Se propone que sería la correspondencia, pues fue una vía de comunicación funcional y recurrida en la época. Hace unos años se publicó un libro con la correspondencia de la teórica marxista: Cartas de Rosa Luxemburgo, en un extracto de una de éstas, remitida a León Jogiches ella le escribe:
«A veces pienso que lo mejor sería que nos viéramos lo menos posible, a veces me transporta un impulso y quiero olvidar todo, arrojarme en tus brazos y llorar [...] el odio me sube y quiero hacerte mal, herirte, mostrarte que no tengo necesidad de tu amor, que podría pasarme sin ti y de nuevo me atormento y me torturo y así todo reco- mienza otra vez» (Argote en El mundo, 2011).

Por otra parte, cuando Avelina Gallegos Gallegos (ex integrante de Las Rosas) es asesinada de un tiro en la cabeza durante una triple expropiación bancaria, supuestamente llevaba consigo una carta escrita por ella para Gilberto Montaño León, alias el Chicano. No se pudo localizar la versión original, por lo que no es posible asegurar que en efecto haya existido dicha carta. Sin embargo, y con la buena voluntad de que ella misma redactó el texto publicado, ${ }^{36}$ éste bien merece algunas observaciones. Al inicio ella le dice a Gil: «Te quiero, mas no puedo seguir adelante. Las fuerzas que tu amor me dan, han sufrido fuertes tormentas y me van debilitando para seguir adelante». Una vez dentro del grupo armado, y con el plan que se tenía luego del triple asalto, Avelina pasaría por completo a la vida clandestina. Mientras tanto: ¿asumía como debilidad un amor que antes le daba fuerzas? ¿O debía comenzar a acatar los nuevos estereotipos de una mujer guerrillera, en

35 Esto es aún más tangible en el hecho de que algunas de las personas muertas y participantes en el triple asalto bancario fueron reconocidas, como el cuerpo de Avelina Gallegos que fue reconocido por el ministerio público que fue a la morgue.

36 Javier H. Contreras, Los informantes. Documentos confidenciales de la guerrilla en Chihuahua, Chihuahua, Textos universitarios, Universidad Autónoma de Chihuahua, 2007. 
los que las expresiones de amor no son permitidas, ni promovidas, ni soportadas por la vida azarosa? Por otra parte, los sentimientos encontrados, casi antagónicos, se asemejan de cierta forma a los de la teórica marxista ¿Cuánto más nos falta por construir alrededor de las subjetividades en el pasado de los movimientos sociales, armados o no, para reconstruir una historia de las relaciones humanas que apunte a potenciar a las personas en lugar de limitarlas?

¿Y cómo delimitar si estas mismas subjetividades no son permitidas en la investigación histórica que busca ser rigurosa y eminentemente posible?: Que el fuego del pasado nos haga arder la piel en el presente. 


\section{REFERENCIAS BIBLIOGRÁFICAS}

Argote, A. (15 de junio de 2011): La comunista enamorada en El mundo, Madrid. Disponible en: http://www.elmundo.es/elmundo/2011/06/15/cultura/1308127736.html

Contreras, J. (2007): Los informantes. Documentos confidenciales de la guerrilla en Chihuahua, Textos universitarios, Universidad Autónoma de Chihuahua, México.

Centro de Estudios Nacionales, S.C. (1964): La campaña electoral de Gustavo Díaz Ordaz. Su gira por los estados de Coahuila, Chihuahua y Sinaloa, Testimonios de una campaña electoral. Ediciones del Centro de Estudios Nacionales, México.

Decreto No. 171 (8 de diciembre de 1954): Periódico Oficial de Gobierno del Estado No. 98, Chihuahua, México.

Demografía (18-24 de junio de 1981): Contextos. La noticia en la prensa mundial. Año 2, No. 24., México.

Dirección General de Estadística (1963): Anuario Estadístico de los Estados Unidos Mexicanos 1960-1961. Secretaria de Industria y Comercio, México.

Dirección General de Estadística (1973): Anuario Estadístico de los Estados Unidos Mexicanos 1970-1971. Secretaría de Industria y Comercio, México.

Dunayevskaya, R. (1985): Rosa Luxemburgo, la liberación femenina y la filosofía marxista de la revolución, Fondo de Cultura Económica, México.

[Nota periodística] El Heraldo de Chihuahua (9 marzo de 1967): México.

García Aguirre, A. (2012): Normalistas y maestros en el movimiento campesino y guerrillero de Chihuahua, 1960-1968. Experiencias de solidaridad y relaciones reticulares en la formación de un sujeto político. Centro de Investigación y de Estudios Avanzados, Departamento de Investigaciones Educativas, México.

Instituto Nacional de Estadística, Geografía e Informática (2006): Indicadores sociodemográficos de Chihuahua (1930 a 2000), México.

Viramontes, O. (12 de octubre de 2009): "Un incidente que hizo temblar a Chihuahua", en El Heraldo de Chihuahua, México. Recuperado de: http://tramoyam1.blogspot. com/2009/10/cronicas-urbanas-chihuahuenses-un.html

\section{ENTREVISTAS}

Entrevista a Irma Campos Madrigal realizada por Nithia Castorena Sáenz el 14 de octubre de 2008. Irma fue fundadora e integrante de la Sociedad Femenil Rosa Luxemburgo e integrante de las redes urbanas de apoyo del Grupo Popular Guerrillero.

Entrevista a Carlos Fernández Baca realizada por Nithia Castorena-Sáenz el 23 de febrero de 2013, en Chihuahua, Chihuahua. Carlos fue integrante de la Sociedad Ignacio Ramírez.

Entrevista a Jaime García Chávez, realizada por Nithia Castorena-Sáenz el 7 de abril de 2013. Jaime fue novio (y posteriormente esposo) de Irma Campos, integrante de la 
Sociedad Ignacio Ramírez e integrante de las redes urbanas de apoyo del Grupo Popular Guerrillero, y colaborador del Grupo Popular Guerrillero “Arturo Gámiz”.

Entrevista a Cristina González Tejeda, realizada por Nithia Castorena Sáenz el 23 de abril de 2013 en Chihuahua, Chihuahua. Cristina fue integrante de la Sociedad Femenil Rosa Luxemburgo e integrante de las redes urbanas de apoyo del Grupo Popular Guerrillero.

Entrevista a Sergio Granados Pineda, realizada por Nithia Castorena-Sáenz el 2 de abril de 2013 en Chihuahua, Chihuahua. Sergio fue integrante de la Sociedad Ignacio Ramírez.

Entrevista a Carmen Monares realizada por Nithia Castorena-Sáenz el 4 de mayo de 2013. Carmen fue integrante del Movimiento de Acción Revolucionaria e invitada por Avelina Gallegos a una supuesta reconfiguración de la Sociedad Femenil Rosa Luxemburgo.

Entrevista a Víctor Orozco Orozco realizada por Nithia Castorena-Sáenz el 3 de abril de 2013, Ciudad Juárez, Chihuahua. Víctor fue parte de la Sociedad Ignacio Ramírez e integrante de las redes urbanas de apoyo del Grupo Popular Guerrillero.

Entrevista a Marco Rascón Córdoba, realizada por Nithia Castorena-Sáenz el 11 de enero del 2012 en Chihuahua, Chihuahua. Marco fue estudiante de la Universidad de Chihuahua, y fue parte del Grupo N o Los Guajiros. 